\title{
Voltage Differencing Current Conveyor Based Linearly Controllable Quadrature Oscillators
}

\author{
R. Sotner, J. Jerabek, J. Petrzela \\ Faculty of Electrical Engineering and \\ Communication \\ Brno University of Technology \\ Czech Republic \\ sotner@feec.vutbr.cz
}

\author{
T. Dostal \\ Dept. of Technical Studies \\ College of Polytechnics Jihlava \\ Jihlava \\ tomas.dostal@vspj.cz
}

\begin{abstract}
This paper presents two new applications of modified version of the voltage differencing current conveyor (VDCC) active device in electronically linearly controllable quadrature oscillators. In this particular case, VDCC element is modified in such a way that it uses electronically adjustable current gain controlled independently in each of output current branches of this device. Brief Spice simulation tests based on emulators of VDCC employing commercially available subparts and including system for amplitude stabilization are also presented. Results confirmed expected behavior.
\end{abstract}

Keywords- electronic control; transconductance; current gain; input resistance; quadrature oscillator; linear tuning; voltage differencing current conveyor

\section{INTRODUCTION}

This study is focused on special modification of voltage differencing current conveyor (VDCC) - socalled controlled gain VDCC (CG-VDCC), where electronic control of current gain of the second internal subpart (electronically controllable current conveyor of second generation - ECCII [2]-[4]) is divided to two independent output branches. Except control of current gain, CG-VDCC offers also two other controllable features - transconductance [5] and resistance of current input terminal [6]. In comparison to all recent works, external utilization (interconnection) of $\mathrm{X}$ input terminal is not supposed in this work. More information about current conveyor-based derivations of active devices can be found in [7]. Dual independent control of gains of output currents allows special features for example in applications as linearly controllable oscillators.

Not many oscillators based on VDCC device with its controllable parameter has been reported in the past. Work [8] deals with single ZC-CG-VDCC (Zcopy feature [1]) element-based oscillator producing output signals with pi/4 phase distance. Parameters $R_{\mathrm{X}}$ and $g_{\mathrm{m}}$ are used for linear control of frequency of oscillations (FO) and single current gain $(B)$ is used for control of condition of oscillations $(\mathrm{CO})$. Circuitry presented in [8] has similar complexity as solutions discussed in this paper. However, it is not suitable for production of quadrature signals. Similarly complex

Research described in this paper was financed by Czech Ministry of Education in frame of National Sustainability Program under grant LO1401. For research, infrastructure of the SIX Center was used. Research described in the paper was supported by Czech Science Foundation projects under No. 14-24186P. single active element-based quadrature oscillator solution was presented in [9]. However, oscillator in [9] utilizes only one electronically controllable parameter $\left(g_{\mathrm{m}}\right)$ and does not offer such variability $(\mathrm{CO}$ and FO are adjustable only by variation of values of external grounded passive components) and FO is not tunable linearly. The work, dealing with VDCC used in oscillators, was published also by Rawat et al. [10]. Similarly as in [9], VDCC concept employs only $g_{\mathrm{m}}$ as adjustable parameter (but not used in application) and resulting structures of oscillators are very complex (two and more active devices). It is however not necessary in many cases.

\section{CG-VDCC BASED OSCILLATORS}

We designed two solutions of quadrature oscillators based on single CG-VDCC active device. Their specifications focus on simple electronic linear controllability of FO, unchangeable output amplitudes during the process of tuning of FO and implementation of precise system for amplitude stabilization (automatic gain control circuit - AGC). Specialty that was not used in previous designs and is typical in both presented solutions in this paper consists in VDCC concept enhanced by two independently controllable output branches of $\mathrm{Z}$ terminal ( $2 \mathrm{x} \mathrm{zn}$, or $\mathrm{zn}$ and $\mathrm{zp}$ terminals).

\section{A. Brief $C G-V D C C$ description}

The CG-VDCC device has been introduced in [11]-[13] (see for more details). Brief principle is indicated in Fig. 1. There are four controllable parameters available (transconductance $-g_{\mathrm{m}}$; resistance of current input terminal $-R_{\mathrm{X}}, \mathrm{X}$ terminal not used in this particular case (grounded); current gains of two output terminals $-B_{1}, B_{2}$ ). We suppose $R_{\mathrm{X}}$ as an "internal" electronically adjustable component. Due to the used concept (as explained later in Fig. 3 and Fig. 5 - division of current to two branches) of the controlled gains in both output branches ( $\mathrm{zp}, \mathrm{zn})$, transfers are defined as $I_{\mathrm{zn}}=$ $0.5 B_{1} I_{\mathrm{X}}, I_{\mathrm{zp}}=0.5 B_{2} I_{\mathrm{X}}$.

\section{B. The first type of the oscillator}

The first designed circuit is shown in Fig. 2. We can see that all controllable features of the CG-VDCC are engaged in order to control specific features of the oscillator. 


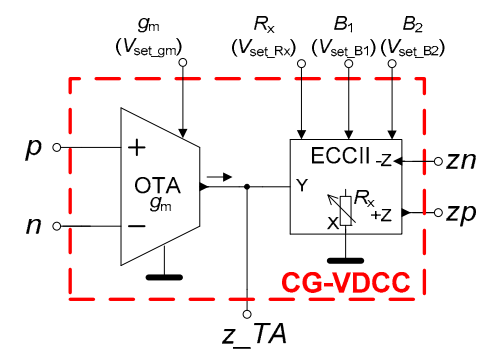

a)

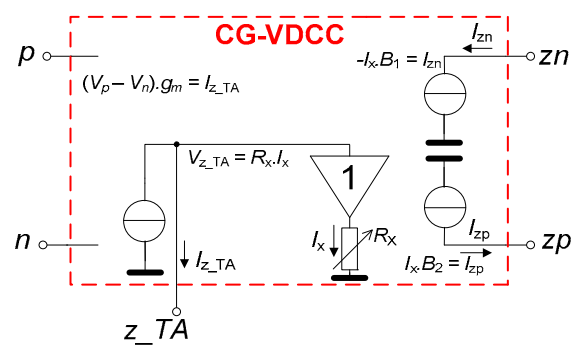

b)

Figure 1. Ideal operation of the CG-VDCC element: a) twosubpart-based concept, b) general behavioral model

In this case, characteristic equation has form:

$$
s^{2}+\frac{\left(2 g_{m} R_{X}-B_{1}\right)}{2 R_{X} C_{1}} s+\frac{g_{m} B_{2}}{2 R_{X} C_{1} C_{2}}=0,
$$

where $\mathrm{CO}$ and $\mathrm{FO}$ can be expressed as:

$$
B_{1} \geq 2 g_{m} R_{X}, \quad \omega_{0}=\sqrt{\frac{g_{m} B_{2}}{2 R_{X} C_{1} C_{2}}} .
$$

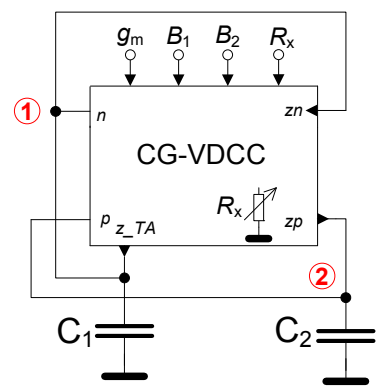

Figure 2. The first type of the quadrature oscillator based on CGVDCC with outputs of two polarities

When comparing (2) and (3), we can see that $\mathrm{CO}$ and FO are mutually dependent in case of using $g_{\mathrm{m}}$ or $R_{\mathrm{X}}$ for tuning. However, current gains can be used for tuning $\left(B_{2}\right)$ and adjusting of $\mathrm{CO}\left(B_{1}\right)$. We can calculate voltage transfer between nodes 2 and 1 as:

$$
V_{2}=-\frac{1}{2} \frac{B_{2}}{s C R_{X}} V_{1}
$$

$B_{2}$ influences amplitude relation between $V_{1}$ and $V_{2}$ that can be expressed (supposing $R_{\mathrm{X}}=1 / g_{\mathrm{m}}=R$ and $\left.C_{1}=C_{2}=C\right)$ as $V_{2}=j \sqrt{ }\left(2 B_{2}\right) V_{1}$. The advantage of this form of CO (2) is in term of product of $g_{\mathrm{m}}$ and $R_{\mathrm{X}}$. In fact, if we suppose $g_{\mathrm{m}}=1 / R_{\mathrm{X}}$, this term disappears from (2) but adjusting of $\mathrm{CO}$ is still available by $B_{1}$ which is beneficial. Simultaneous control of $R_{\mathrm{X}}$ and $g_{\mathrm{m}}$ can be used for linear control of FO $\left(B_{2}=2\right.$ for unity gain to compensate 2 in denominator of (3)) without disturbance of $\mathrm{CO}$ and it ensures constant amplitudes of produced signals during the tuning process:

$$
V_{2}=-\frac{1}{s C_{2} R_{X}} V_{1} \Rightarrow\left|\begin{array}{l}
R_{X}=1 / g_{m}=R \\
C_{1}=C_{2}=C
\end{array}\right| \Rightarrow V_{2}=j V_{1} .
$$

Therefore, we have three possible parameters suitable for FO control: $B_{2}$ only; simultaneous adjusting of $R_{\mathrm{X}}$ and $g_{\mathrm{m}}$; both ways together in order to extend FO range. Note that the last approach is not so beneficial because it causes problems with one amplitude that is dependent on FO. Fig. 3 shows particular behavioral implementation with macromodels of commercially available devices forming the first solution of oscillator presented in Fig. 2.

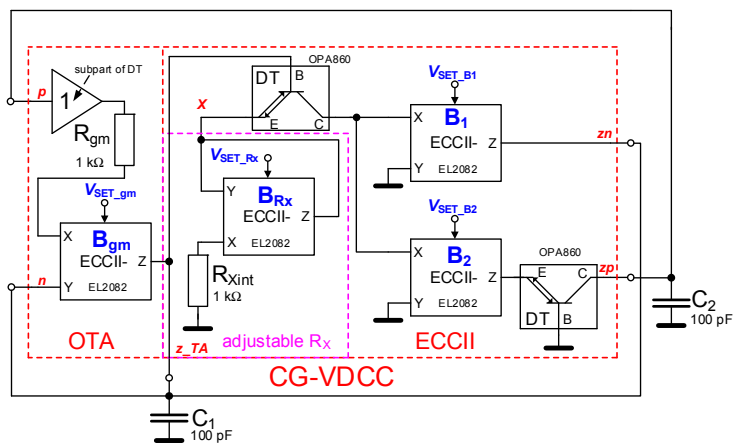

Figure 3. The first type of the oscillator based on behavioral emulator of the CG-VDCC device with differential outputs

We decided to use the second variant of the FO control (linearly by $g_{\mathrm{m}}=1 / R_{\mathrm{X}}$ ) for verification. Utilization of electronically controllable current conveyors of second generation (ECCII) available under designation EL2082 and subparts of so-called "diamond transistor" (DT) OPA860 (including diamond transistor and diamond voltage buffer in one package) were utilized to provide four electronically controllable parameters used for control of the device and therefore also its applications. These parameters are formed as follows: $g_{\mathrm{m}}=B_{\mathrm{gm}} / R_{\mathrm{gm}} \cong V_{\mathrm{SET} \_\mathrm{gm}} / R_{\mathrm{gm}}$; $R_{\mathrm{X}}=B_{\mathrm{RX}} / R_{\text {Xint }} \cong V_{\text {SET_Rx }} / R_{\text {Xint }} ; \quad B_{1} \cong V_{\text {SET_B1}} ;$ $B_{2} \cong V_{\text {SET_B2}}$. Taking previously discussed simplifications of the oscillator design into account, we can directly specify equation for FO, where DC control voltages $V_{\text {SET }}$ are included:

$$
\omega_{0}=\sqrt{\frac{g_{m} B_{2}}{2 R_{X} C_{1} C_{2}}} \cong \sqrt{\frac{V_{S E T_{-} g m} V_{S E T_{-} R x} V_{S E T_{-} B 2}}{2 R_{g m} R_{X_{\mathrm{int}} C_{1} C_{2}}}} .
$$

Condition of oscillation ( $g_{\mathrm{m}}=1 / R_{\mathrm{X}}=R$ is fulfilled) has now form: $V_{\text {SET B1 }} \geq 2$. In case of $V_{\text {SET B2 }}=2 \mathrm{~V},(6)$ simplifies to:

$$
\omega_{0} \cong \sqrt{\frac{V_{S E T_{-} g m} V_{S E T_{-} R x}}{R_{g m} R_{X \mathrm{int}} C_{1} C_{2}}} \cong \frac{V_{S E T_{-} F O}}{R C},
$$

and linear control of FO is possible $\left(V_{\text {SET_gm }}=V_{\text {SET_B2 }}=V_{\text {SET_FO }}\right)$.

\section{The second type of the oscillator}

Despite influence of term $g_{\mathrm{m}} R_{\mathrm{X}}$ in $\mathrm{CO}$ (2) can be removed by utilization of $g_{\mathrm{m}}$ and $R_{\mathrm{X}}\left(g_{\mathrm{m}}=1 / R_{\mathrm{X}}\right)$ for 
linear tuning of FO, the CG-VDCC device can be used also for design of the quadrature oscillator without this term $\left(g_{\mathrm{m}} R_{\mathrm{X}}\right)$ present in $\mathrm{CO}$. The circuit solution fulfilling these requirement is shown in Fig. 4. It contains additional grounded resistor in comparison to Fig. 2. CG-VDCC with two current outputs having the same polarity but independently controllable current gains was used in this case. Figure 5 shows the behavioral model of the second type of the oscillator and it is much simpler than model in Fig. 3.

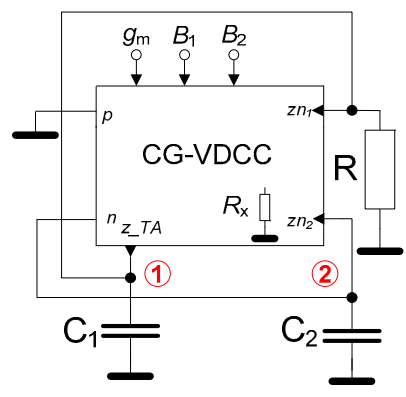

Figure 4. The second type of the quadrature oscillator based on CG-VDCC with outputs having the same polarity

The oscillator in Fig. 4 has characteristic equation in form:

$$
s^{2}+\frac{\left(2 R_{X}-R B_{1}\right)}{2 R_{X} R C_{1}} s+\frac{g_{m} B_{2}}{2 R_{X} C_{1} C_{2}}=0,
$$

where FO has the same form as (3) and $\mathrm{CO}$ is expressed as: $B_{1} \geq 2 R_{\mathrm{X}} / R$. For equality $R_{\mathrm{X}}=R$, we obtain $B_{1} \geq 2$.

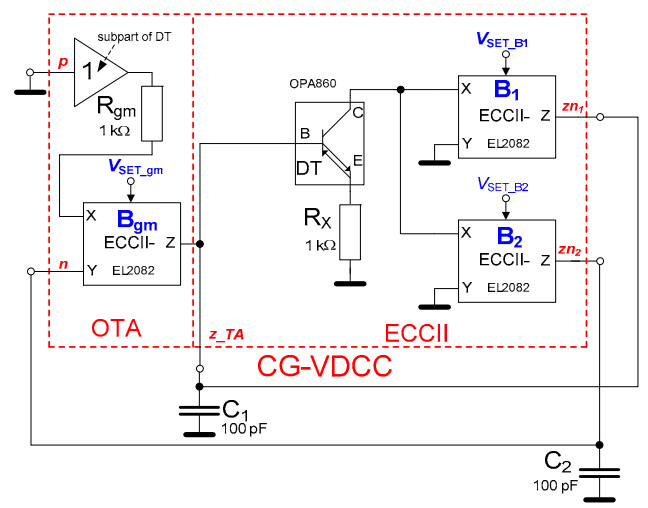

Figure 5. The second type of the oscillator based on behavioral emulator of the CG-VDCC device with two negative outputs

Taking real DC control voltages into account, FO can be transformed to:

$$
\omega_{0}=\sqrt{\frac{g_{m} B_{2}}{2 R_{X} C_{1} C_{2}}} \cong \sqrt{\frac{V_{S E T_{-} g m} V_{S E T_{-} B 2}}{2 R_{g m} R_{X} C_{1} C_{2}}} \cong \frac{V_{S E T_{-} F O}}{\sqrt{2} R C_{2}} .
$$

Supposing $R_{\mathrm{X}}=R, V_{\mathrm{SET} \text { gm }}=V_{\mathrm{SET} \text { B } 2}=V_{\mathrm{SET}+\mathrm{FO}}-$ used for linear control of $\mathrm{FO}, C_{1}=C_{2}=C$, resulting relation of produced output signals has form:

$$
V_{2}=-\frac{1}{2} \frac{1}{s C_{2} R_{X}} V_{1} \Rightarrow\left|\begin{array}{l}
R_{X}=R \\
C_{1}=C_{2}=C
\end{array}\right| \Rightarrow V_{2}=j \frac{\sqrt{2}}{2} V_{1} .
$$

\section{Amplitude stabilization}

Stabilization of amplitude of produced signals ensures constant output level without fluctuations and decreases THD. Inertial system for automatic gain control (AGC) in loop can be established as shown in Fig. 6.

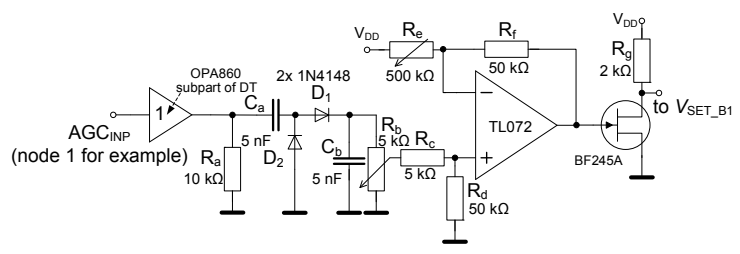

Figure 6. AGC system for amplitude stabilization

\section{RESULT OF VERIFICATION}

Both oscillators shown in Fig. 3 and Fig. 5 together with AGC from Fig. 6 were analyzed using PSpice software. Initial design of both solutions supposes values $R_{\mathrm{X}}=R_{\mathrm{gm}}=R=1 \mathrm{k} \Omega, C_{1}=C_{2}=C=100 \mathrm{pF}$.

\section{A. The first type of the oscillator}

The first type of the circuit (Fig. 3) supposes $B_{2}=2\left(V_{\text {SET_B}}=2 \mathrm{~V}\right)$. All necessary constants are included directly in figures. Tuning of the oscillator in frequency domain is shown in Fig. 7. Results indicate the THD below 1\% (suppression of levels of higher harmonic components from fundamental tone is more than $45 \mathrm{dBc})$. Dependence of FO on $V_{\mathrm{SET} \_\mathrm{gm}}=V_{\mathrm{SET} \text { Rx }}$ and dependence of produced levels $\left(V_{\mathrm{p}-\mathrm{p}}\right)$ on FO are shown in Fig. 8 and Fig. 9. Oscillator is tunable approximately from $0.3 \mathrm{MHz}$ to $2.6 \mathrm{MHz}$ $\left(V_{\text {SET_gm }}=V_{\text {SET_Rx }}\right.$ from 0.2 to $\left.2 \mathrm{~V}\right)$ while levels of output voltages are between 1.8 and $2 \mathrm{~V}_{\mathrm{p}-\mathrm{p}}$.

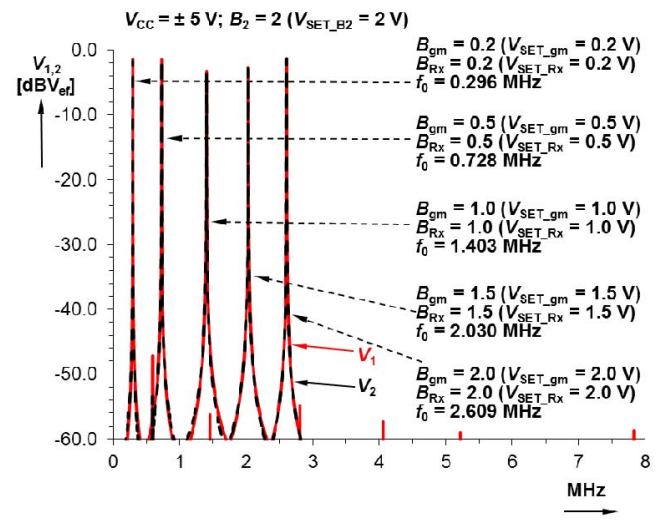

Figure 7. Spectral analysis of produced signals for five discrete frequencies (first oscillator)

\section{B. The second type of the oscillator}

The same evaluation as in previous case was provided for the second oscillator. Spectral purity (Fig. 10) of produced signals is slightly worse in direction to the lowest frequencies (separation about $40 \mathrm{dBc}-\mathrm{THD}$ about $1 \%$ ). Tunability range is slightly moved to lower frequencies (from $0.2 \mathrm{MHz}$ to $1.9 \mathrm{MHz}$ for the same range of DC control voltage as in previous case) in comparison to previous results, due to difference of (7) and (9), see Fig. 8. Small variations of the output levels are indicated in Fig. 9. Setting of AGC influences obtained results and in case 
of real experiments we can expect better results because of simpler setting of AGC in case of real implementation.

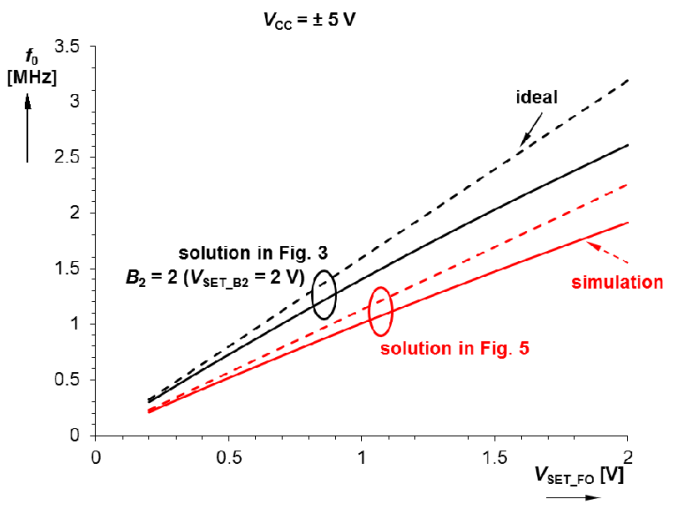

Figure 8. Dependence of FO on DC control voltage (both oscillators)

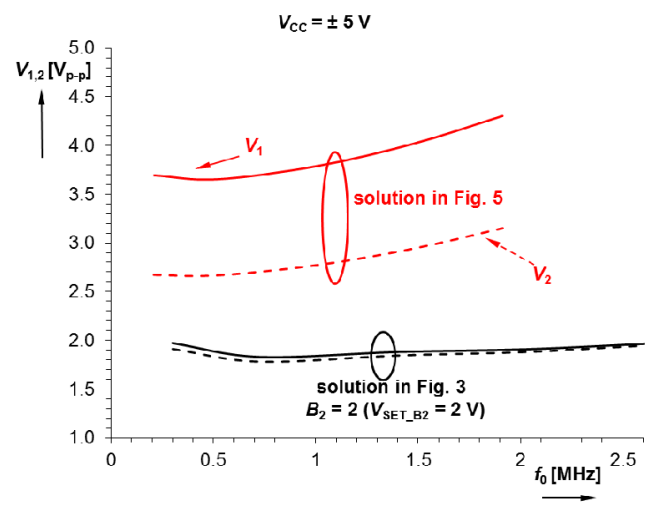

Figure 9. Dependence of output levels on FO (both oscillators)

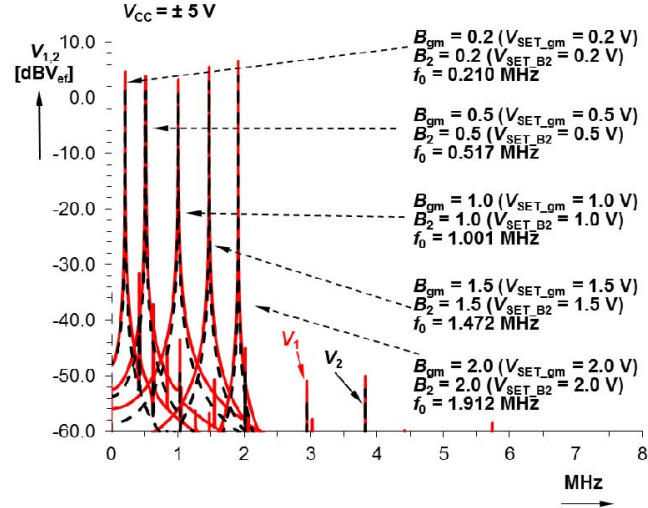

Figure 10. Spectral analysis of produced signals for five discrete frequencies (second oscillator)

\section{CONCULSION}

We prepared two solutions of oscillators based on CG-VDCC device. Both solutions are suitable for linear control of FO and independent control of CO. Tuning ranges are different because of difference of equations for $\mathrm{FO}$ (the second variant has slightly decreased band due to constant 2 in denominator of theoretical FO). No that this disadvantage can be minimized if $V_{\text {SET_gm }}=2 V_{\text {SET_B2 }}$ (or vice versa).

\section{REFERENCES}

[1] D. Biolek, R. Senani, V. Biolkova, Z. Kolka, "Active Elements for Analog Signal Processing: Classification, Review, and New Proposals," Radioengineering, vol. 17, no. 4, pp. 15-32, 2008

[2] W. Surakampontorn, W. Thitimajshima, "Integrable electronically tunable current conveyors," IEE Proc.-G, vol. 135, no. 2, pp. 71-77, 1988.

[3] A. Fabre, N. Mimeche, "Class A/AB second-generation current conveyor with controlled current gain," Electronics Letters, vol. 30, no. 16, pp. 1267-1268, 1994.

[4] S. Minaei, O. K. Sayin, H. Kuntman, "A new CMOS electronically tunable current conveyor and its application to current-mode filters," IEEE Trans. on Circuits and Systems $I$, vol. 53, no. 7, pp. 1448-1457, 2006.

[5] R. L. Geiger, E. Sanchez-Sinencio, "Active filter design using operational transconductance amplifiers: a tutorial," IEEE Circ. And Devices Magazine, vol. 1, pp. 20-32, 1985.

[6] A. Fabre, O. Saaid, F. Wiest, C. Boucheron, "High frequency applications based on a new current controlled conveyor," IEEE Trans. on Circuits and Systems - I, vol. 43, no. 2, pp. $82-91,1996$.

[7] R. Senani, D. R. Bhaskar, A. K. Singh, Current Conveyors: Variants, Applications and Hardware Implementations, Springer International Publishing, 560 pages, 2015.

[8] R. Sotner, J. Jerabek, J. Petrzela, N. Herencsar, R. Prokop, K. Vrba, "Second-order Simple Multiphase Oscillator Using ZCopy Controlled-Gain Voltage Differencing Current Conveyor," Elektronika Ir Elektrotechnika, vol. 20, no. 9, pp. 13-18, 2014.

[9] D. Prasad, D. R. Bhaskar, M. Srivastava, "New Signle VDCC-based Explicit Current-Mode SRCO Employing All Grounded Passive Components," ELECTRONICS, vol. 18, no. 2, pp. 81-88, 2014.

[10] M. Rawat, M. Bansal, "Voltage Mode Two-Phase and FourPhase Sinusoidal Oscillator Using VDCC," International Journal of Advanced Research in Electronics and Communication Engineering, vol. 4, no. 6, pp. 1687-1691, 2015.

[11] R. Sotner, N. Herencsar, J. Jerabek, R. Prokop, A. Kartci, T. Dostal, K. Vrba, "Z-Copy Controlled-Gain Voltage Differencing Current Conveyor: Advanced Possibilities in Direct Electronic Control of First-Order Filter," Elektronika Ir Elektrotechnika, vol. 20, no. 6, pp. 77-83, 2014.

[12] R. Sotner, J. Jerabek, N. Herensar, T. Dostal, K. Vrba, "Design of Z-copy controlled- gain voltage differencing current conveyor based adjustable functional generator," Microelectronics Journal, vol. 46, no. 2, pp. 143-152, 2015.

[13] R. Sotner, N. Herencsar, J. Jerabek, K. Vrba, T. Dostal, W. Jaikla, B. Metin, "Novel first-order all-pass filter applications of z- copy voltage differencing current conveyor," Indian Journal of Pure and Applied Physics, vol. 53, no. 8, pp. 537$545,2015$. 